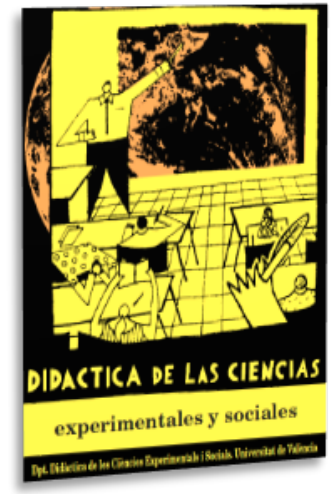

\title{
Enfoque epistemológico y experimental en la enseñanza de las ciencias, una estrategia didáctica para el aprendizaje de la teoría de la luz de Newton
}

\author{
Epistemological and experimental approach in \\ science teaching, a didactic strategy for \\ learning Newton's theory of light
}

DOI: $10.7203 / D C E S .41 .20806$

\author{
Sergio J. Vargas-Vargas \\ Universidad Católica de Manizales (Colombia), sergio.vargas3@ucm.edu.co \\ ORCID iD: https://orcid.org/0000-0001-6273-6513 \\ Luis H. Carmona-Ramírez \\ Universidad Católica de Manizales (Colombia), lucarmona@ucm.edu.co \\ ORCID iD: https://orcid.org/0000-0002-4136-851X
}

\begin{abstract}
RESUMEN: La controversia que se dio en el siglo XVII en torno a la naturaleza de la luz y la formación de los colores por refracción es un interesante ejemplo de cómo se dan las revoluciones en la conceptualización de un fenómeno y del carácter revelador que puede tener un experimento. El objetivo de este estudio consistió en implementar una estrategia para el fortalecimiento del aprendizaje de la teoría de la luz de Newton a partir de la experimentación y la epistemología. La muestra la conformaron 15 estudiantes, a los que se les realizó una prueba inicial y una prueba final, después de aplicado el tratamiento con actividades experimentales. Los resultados evidenciaron cómo los educandos mejoraron en sus desempeños académicos, mostrando que la estrategia pedagógica favorece la aprehensión de la teoría de la luz, convirtiéndose en un interesante recurso pedagógico para la enseñanza de contenidos de carácter científico.
\end{abstract}

Palabras ClaVe: teoría de la luz, experimento, epistemología de la ciencia, enseñanza de la Física

ABSTRACT: The controversy that arose in the 17th century around the nature of light and the formation of colors by refraction is an interesting example of how revolutions occur in the conceptualization of a phenomenon and of the revealing character that an experiment can have. The objective of this study was to implement a strategy to improve the learning of Newton's theory of light through experimentation and epistemology. The sample consisted of 15 students, who underwent an initial test and a final test, after applying the sequence with experimental activities. The results showed how the students improved in their academic performance, showing that the pedagogical strategy favors the apprehension of the theory of light, becoming an interesting pedagogical resource for teaching scientific content.

KEYWORDS: theory of light, experiment, science epistemology, Physics teaching

Fecha de recepción: junio de 2021 Fecha de aceptación: diciembre de 2021 


\section{INTRODUCCIÓN}

En el año de 1666, Sir Isaac Newton escribió una carta a la Royal Society para comunicar su teoría acerca de la luz en la que narraba lo siguiente:

A tal efecto dejé mi cuarto en la oscuridad e hice un pequeño agujero en el postigo para que entrara una adecuada cantidad de luz del sol. Coloqué mi prisma junto al agujero para que la luz se refractara hacia la pared opuesta del cuarto. Al principio fue una diversión muy agradable ver los colores vivos e intensos así producidos. Pero después de un rato me puse a considerarlos de una manera más prudente y me asombró ver que tenían una forma oblonga, aunque según las leyes aceptadas de la refracción esperaba que fueran circulares (Newton, 1666, como se citó en Lafuente, Valverde, y Pimentel, 2004, p. 51).

La anterior descripción nos muestra, como el experimento, se convierte en un foco de aprendizaje, ya que el hecho de conjeturar algo y observar un resultado diferente, permite redescubrir en esencia el principio de una obra. Es así como la enseñanza de la ciencia en la escuela y particularmente de la física, se ha convertido en una revolución que está pendiente por darse, pues es necesario implementar el experimento como una herramienta didáctica para problematizar la construcción de las teorías y no a manera de evidenciar la concordancia entre lo predicho por una ecuación y el devenir de un fenómeno natural. En el ámbito del discurso educativo se reconoce la importancia del experimento en el aprendizaje de la ciencia, no sólo como una herramienta que permite a los estudiantes tener contacto con los fenómenos que aborda la teoría, sino como un mecanismo que facilita el aprendizaje y que además debe ser entendido como un elemento que permite falsear o validar parcialmente una teoría. Sin embargo, en la escuela el experimento se desnaturaliza, al tiempo que se cercena su potencial como mediador en la enseñanza y el aprendizaje de la ciencia y el método científico.

El experimento pierde su carácter pedagógico y didáctico si no se entiende dentro de un contexto histórico en el que fue determinante, en el que permitió discernir entre dos o más modelos teóricos (Morcillo, 2016; Monroy, 2016). Al respecto Solbes y Sinarcas (2009), le conceden una "gran importancia al desarrollo histórico de las ciencias, no tanto por "contar" la historia del tema tratado" (p. 147) sino por extraer de la misma los problemas más significativos y colocar al estudiante en situación de poder abordar y resolver cada situación planteada. Así mismo, Matthews (1991) le hace una crítica al modelo tradicional de la enseñanza de las ciencias, dándole un rol muy significativo al papel que desempeñan los filósofos y los historiadores de la ciencia en la enseñanza y a los beneficios que estos aportan al aprendizaje científico.

Ahora bien, el desarrollo de las teorías de la física clásica y moderna se da por una confrontación de ideas que la enseñanza debe evidenciar; revelar el debate que se da en torno a la aceptación o rechazo de un modelo, permite a los estudiantes entender la ciencia como un proceso social, como una construcción de los seres humanos, como inacabada.

En la escuela la enseñanza de la óptica consiste en abordar un conjunto de fenómenos asociados al comportamiento de la luz, entre los que se incluyen la reflexión, la refracción, la interferencia y la polarización, presentar el conjunto de ecuaciones y conceptos que conforman el modelo teórico aceptado actualmente, resolver un conjunto de problemas que abarcan todas las situaciones en las que el modelo funciona y realizar prácticas experimentales que tiene como finalidad la reafirmación de la teoría, pero se ha olvidado la importancia de enseñarla desde la perspectiva histórica, dada la importancia de reconocer la naturaleza de la luz, ya que estos han constituido uno de los fenómenos físicos más "controvertidos a lo largo de la Historia de la Ciencia”(Perales y Nievas, 1991, p. 77). En general la enseñanza de la física en la escuela sigue esta dinámica, se muestra a los estudiantes el último resultado de un proceso de larga duración, pero 
nunca el proceso; este enfoque desfigura los contenidos en cuanto que los desvincula del contexto histórico en el que se desarrollaron y en el que fueron revolucionarios.

Algunas investigaciones como Bravo (2016) y Cepeda (2017) rescatan el rol del experimento en la enseñanza de las ciencias, así como la importancia de la reseña histórica y el papel de la difracción en la evolución de la teoría ondulatoria, así mismo, existen otros trabajos que valoran de manera significativa el uso de la historia y la filosofía de la ciencia en la enseñanza de la física ya que potencia los procesos de enseñanza y aprendizaje en la escuela, además de permitir una discusión permanente de los modelos que alimentaron dicha teoría, permitiendo al maestro y a sus estudiantes comprender desde otra perspectiva la importancia que suscitó dicha experiencia en la época (Castro et al., 2012; Aguilar y Alamino, 2014; Morcillo, 2016; Álvarez y Manzano, 2018; Solbes y Traver 1996, 2001, 2003).

Finalmente, este trabajo pretende mostrar el potencial pedagógico que exhibe este debate histórico y los experimentos con los que Newton reformuló completamente la concepción de la luz como referentes para la enseñanza de la ciencia, además de validar que es más fácil y significativo entender el modelo teórico reciente que describe un fenómeno físico si se presenta en contraste con los paradigmas que tenían vigencia en el pasado, si se entiende el desarrollo histórico de las ideas y las controversias que suscitó en la época la aceptación de la teoría, si se presenta el debate atado a los experimentos que permitieron entender de una nueva forma un comportamiento que se entendía en otros términos, así como mostrar por medio de la enseñanza la formación de los colores por refracción y vincular a esto la discusión acerca de la naturaleza de la luz. Se espera que se convierta en un referente de cómo enseñar física en la escuela secundaria de una forma vivencial, contextualizada y significativa, extrapolando la experiencia particular en óptica a cualquier contenido que se pretenda enseñar y aprender y que, por su esencia como fenómeno natural cuya conceptualización se construyó a partir del método científico, pueda ser aproximado de la misma manera.

\section{Metodología}

La metodología que se utilizó para el desarrollo del trabajo de campo fue la fenomenológica experiencial, ya que se describen las situaciones cotidianas que se presentan en un contexto determinado y se manifiestan cambios o fenómenos, dando importancia a la función y al significado de los actos humanos, valorando la realidad como es vivida y percibida, con las ideas, sentimientos y motivaciones de sus actores. De acuerdo con Íñiguez (1999) "La cuantificación y medición de procesos tales como opiniones, creencias, actitudes, valores, hábitos, comportamientos y otros se ha presentado como uno de los avances más importantes, y se ha convertido en el principal indicador y criterio de desarrollo científico" (p. 496). La anterior afirmación permite el uso de datos cuantitativos para poder medir las percepciones sin rivalizar con los métodos puristas $\mathrm{o}$ cuantificables, por otro lado la fenomenología desde el punto de vista metodológico "intenta descubrir todo aquello que aparece como pertinente y significativo en las percepciones, sentimientos y acciones de los actores sociales" (Bisquerra, 2009, p. 317). La población objeto de estudio la conformaron 15 estudiantes de grado $10^{\circ}$ y $11^{\circ}$ de la Institución educativa el Madroño del municipio de Belálcazar Caldas - Colombia, dicha institución es de carácter rural, y su población está conformada por familias de estrato social 0,1 y 2, dicha estratificación corresponde a grupos de familias con recursos económicos bajos.

Se partió por lo tanto de una unidad de análisis, a la cual se le denominó Naturaleza de la Luz. En la Tabla 1, se muestra la relación con las respectivas categorías y los instrumentos utilizados. Posteriormente se realizó la operacionalización de la variable de estudio. En este caso la variable utilizada fue de carácter nominal ya que el enfoque de la investigación es cualitativo, en la tabla 2 se puede observar la forma como se dispuso dicha operacionalización. 
TABLA 1. Unidad de análisis

\begin{tabular}{ccc}
\hline Unidad de Análisis & Categorías & Instrumento \\
& $\begin{array}{c}\text { Formación de colores a partir de la } \\
\text { luz blanca }\end{array}$ & $\begin{array}{c}\text { Guía de laboratorio \# 1 } \\
\text { Pretest - Postest }\end{array}$ \\
\cline { 2 - 3 } Naturaleza de la luz & $\begin{array}{c}\text { Clasificación de los colores de la } \\
\text { luz }\end{array}$ & $\begin{array}{c}\text { Guía de laboratorio \# 2 } \\
\text { Pretest - Postest }\end{array}$ \\
\cline { 2 - 3 } & Mezclas de colores & $\begin{array}{c}\text { Guía de laboratorio \# 3 } \\
\text { Pretest - Postest }\end{array}$ \\
\hline
\end{tabular}

Fuente: elaboración propia

TABLA 2. Diseño de la operacionalización de la variable

\begin{tabular}{|c|c|c|c|c|}
\hline \multicolumn{5}{|c|}{ Operacionalización de las variables } \\
\hline $\begin{array}{l}\text { Variable } \\
\text { nominal }\end{array}$ & $\begin{array}{l}\text { Definición de la } \\
\text { variable }\end{array}$ & Dimensiones & Desempeños & $\begin{array}{l}\text { Técnicas e } \\
\text { instrumentos }\end{array}$ \\
\hline \multirow{3}{*}{$\begin{array}{c}\text { Aprendizaje } \\
\text { de la } \\
\text { naturaleza de } \\
\text { luz y } \\
\text { formación de } \\
\text { colores } \\
\text { desde la } \\
\text { perspectiva } \\
\text { de Newton }\end{array}$} & \multirow{3}{*}{$\begin{array}{c}\text { Apropiación de la } \\
\text { teoría de la luz de } \\
\text { Newton propuesta } \\
\text { en siglo XVII, a } \\
\text { partir de la } \\
\text { utilización de una } \\
\text { propuesta didáctica } \\
\text { basada en la } \\
\text { epistemología de la } \\
\text { ciencia y la } \\
\text { experimentación } \\
\text { con mediación de } \\
\text { WhatsApp. }\end{array}$} & $\begin{array}{c}\text { Cognitiva y } \\
\text { Experimental }\end{array}$ & $\begin{array}{c}\text { Diferencia modelos } \\
\text { para explicar la } \\
\text { naturaleza y el } \\
\text { comportamiento de la } \\
\text { luz. }\end{array}$ & $\begin{array}{l}\text { Pretest, } \\
\text { postest, } \\
\text { Observación } \\
\text { directa }\end{array}$ \\
\hline & & $\begin{array}{l}\text { Cognitiva y } \\
\text { Experimental }\end{array}$ & $\begin{array}{l}\text { Clasifica los colores } \\
\text { de la luz al establecer } \\
\text { su comportamiento en } \\
\text { interacción con el } \\
\text { prisma }\end{array}$ & $\begin{array}{l}\text { Pretest, } \\
\text { postest, } \\
\text { Observación } \\
\text { directa }\end{array}$ \\
\hline & & $\begin{array}{l}\text { Cognitiva y } \\
\text { Experimental }\end{array}$ & $\begin{array}{l}\text { Reconoce que el color } \\
\text { es una cualidad de la } \\
\text { luz y por tanto es } \\
\text { manipulable, en } \\
\text { consonancia con las } \\
\text { ideas propuestas por } \\
\text { Newton }\end{array}$ & $\begin{array}{l}\text { Pretest, } \\
\text { postest, } \\
\text { Observación } \\
\text { directa }\end{array}$ \\
\hline
\end{tabular}

Fuente: elaboración propia

TABLA 3. Rúbrica de evaluación del Prestest y del postest

\begin{tabular}{|c|c|c|c|}
\hline $\begin{array}{r}\text { Bajo } \\
1-1,9 \\
\end{array}$ & $\begin{array}{l}\text { Básico } \\
2-2,9 \\
\end{array}$ & $\begin{array}{c}\text { Alto } \\
3-3,9 \\
\end{array}$ & $\begin{array}{c}\text { Superior } \\
4-5\end{array}$ \\
\hline $\begin{array}{l}\text { Desconoce de manera } \\
\text { general los conceptos } \\
\text { relacionados con la } \\
\text { naturaleza de la luz, } \\
\text { su comportamiento } \\
\text { en interacción con el } \\
\text { prisma y el color } \\
\text { como cualidad de la } \\
\text { misma, en } \\
\text { concordancia con la } \\
\text { teoría propuesta por } \\
\text { Isaac Newton. }\end{array}$ & $\begin{array}{l}\text { Reconoce algunos } \\
\text { conceptos } \\
\text { relacionados con la } \\
\text { naturaleza de la luz, } \\
\text { su comportamiento } \\
\text { en interacción con el } \\
\text { prisma y el color } \\
\text { como cualidad de la } \\
\text { misma, en } \\
\text { concordancia con la } \\
\text { teoría propuesta por } \\
\text { Isaac Newton. }\end{array}$ & $\begin{array}{c}\text { Identifica la mayoría } \\
\text { de conceptos } \\
\text { relacionados con la } \\
\text { naturaleza de la luz, } \\
\text { su comportamiento } \\
\text { en interacción con el } \\
\text { prisma y el color } \\
\text { como cualidad de la } \\
\text { misma, en } \\
\text { concordancia con la } \\
\text { teoría propuesta por } \\
\text { Isaac Newton. }\end{array}$ & $\begin{array}{l}\text { Reconoce claramente } \\
\text { los conceptos } \\
\text { relacionadas con la } \\
\text { naturaleza de la luz, } \\
\text { su comportamiento } \\
\text { en interacción con el } \\
\text { prisma y el color } \\
\text { como cualidad de la } \\
\text { misma, en } \\
\text { concordancia con la } \\
\text { teoría propuesta por } \\
\text { Isaac Newton. }\end{array}$ \\
\hline
\end{tabular}

Fuente: elaboración propia 
Los instrumentos utilizados para la recolección de los datos fueron el pretest, el postest y una secuencia didáctica conformada por tres guías de laboratorios. En la tabla 3 se relaciona la rúbrica empleada para medir los resultados de la prueba del pretest y del postet.

Para el desarrollo de la secuencia de actividades experimentales se tuvieron en cuenta el contexto histórico y experimental de la teoría de la luz de Newton en el siglo XVII, específicamente la naturaleza de la luz y la formación de los colores, para lo cual se recurrió a prototipos experimentales diseñados por los maestros; "esa preocupación por acercar la experimentación mediante el diseño de experimentos de fácil adquisición es todavía hoy una importante cuestión en la enseñanza de las ciencias, y en particular, de la óptica" (Pérez y Falcón, 2009, p. 453).

Dichas actividades permitieron a los estudiantes confrontar sus nociones previas con las ideas de esta época y apropiarse del modelo propuesto. Su diseño se basó en el Modelo Escuela Nueva (Fundación Escuela Nueva, 2021), este modelo pedagógico fue creado en la república de Colombia con la intención de satisfacer las necesidades de cobertura educativa del contexto rural. Es considerada una alternativa que permite el acceso a la educación formal de diversas poblaciones vulnerables en el país, especialmente por su ubicación geográfica, ya que facilita el aprendizaje del estudiante a partir del uso de estrategias que dinamizan el aula. Una de las características más sobresalientes es el respeto al ritmo individual del aprendizaje que permite la flexibilidad frente a la entrega de actividades académicas, igualmente favorece el trabajo en equipo y la autonomía, por lo que los avances dependen en gran medida de la motivación de los estudiantes por gestionar su proceso (López, 2020). La secuencia estaba conformada por tres guías de laboratorios que son mencionadas a continuación:

\subsection{Guía 1. Formación de colores a partir de la luz}

En ella se propuso construir la idea de que la luz blanca es una mezcla de luces de colores, así como que el negro es la ausencia de luz. Se tuvieron en cuenta los preconceptos que los estudiantes tenían con respecto a la mezcla de colores a través de pigmentos para construir la idea de que la luz manifiesta un comportamiento diferente. Así mismo se pretendía mostrar como las ideas de Newton, en contraste con la de Hooke y Descartes fueron innovadoras en tanto le atribuyó especial importancia al experimento como forma de comprobar hipótesis. Se previó que el experimento pudiera desarrollarse con la ayuda de un prisma óptico para poder observar la descomposición de la luz blanca. El alumnado debía desarrollar una guía de laboratorio fundamentada en el modelo de escuela nueva, en el momento inicial denominado vivencia pintaban círculos con colores primarios, posteriomente se les indicó que mezclaran dichos colores y describieran que nuevos colores se generaban, en el segundo momento denominado fundamentación debían leer sobre el contexto histórico y epistemológico de la filosofía natural Newtoniana, el tercer momento se denominó ejercitación, en este a los estudiantes se les asignó la tarea de generación de un espectro al hacer pasar luz blanca por un medio refrangible (prisma) e identificaron los colores que componían dicha luz, el cuarto momento el de la aplicación sirvió para que los estudiantes explicaran el fenómeno dando respuesta a la pregunta: ¿por qué se descompone la luz?, finalmente en el momento de complementación se invitó al alumnado a que en familia construyeran un arco iris empleando la aspersión de gotas de lluvia frente a un haz de luz solar de tal manera que el fenómeno presentado correspondiera a un efecto similar al que sucede cuando se observa el arco iris. La fase experimental se desarrolló de manera particular en la casa de los estudiantes y se dispuso de 3 horas para su elaboración.

\subsection{Guía 2. Clasificación de los colores de la luz}

En esta experiencia se construyó la idea de que los colores en los que se descompone la luz blanca pueden ser refractados nuevamente, tal como lo hizo Newton. Esta experiencia permitió 
evidenciar que los colores fundamentales son el rojo, el verde y azul. Por otro lado y a partir de la experiencia anterior se pudo realizar mezclas aditivas de colores para encontrar los demás. Se pretendía además, mostrar las concepciones relacionadas con la composición de la luz, desde las interpretaciones de la filosofía mecánica frente a la filosofía natural de Newton. De la misma forma que en la guía 1 se dispuso de un laboratorio diseñado con el modelo de escuela nueva y sus respectivos momentos, en la vivencia se les solicitó llenar un recipiente de agua introduciendo dentro de él un espejo y ubicándose en un lugar en el cuál se percibiera la luz solar, posterior a ello debían reflejar la luz hasta que chocara con el espejo dentro del agua, el segundo momento de la fundamentación se invitó por grupos a realizar la lectura del relato en el que Newton expuso los hallazgos de su teoría frente a la Real Society en 1667, el tercer momento de la ejercitación sirvió para que los estudiantes produjeran el espectro al hacer pasar luz blanca de un prisma, luego de colimar cada color del espectro y hacerlo incidir en otro prisma y así determinar si el color seleccionado se descomponía o no en más colores. Por último en la aplicación y la complementación el alumnado trabajaba en familia un test sobre combinación de los colores. La fase experimental se desarrolló de manera particular en la casa de los estudiantes y se dispuso de 3 horas para su elaboración.

\subsection{Guía 3. Mezclas de colores}

El propósito de esta experiencia consistió en reconocer que el color es una cualidad de la luz, por lo tanto, es manipulable y puede producirse en consonancia con las ideas propuestas por Newton. Los objetos absorben algunas frecuencias de onda y reflejan otras, son éstas las que percibimos y dan la idea de su color. Se procuró, en la misma medida, la realización de actividades experimentales que permitieran el descubrimiento, previo a la formalización inicial de los conceptos. Se continuó con el desarrollo de un laboratorio igualmente diseñado bajo el modelo de escuela nueva, en la vivencia el alumnado fue invitado a que diseñaran tres fuentes de luces de colores (rojo, verde y azul) y se les invitó a resolver un reto denominado jugando con los rayos luminosos, en la fundamentación cada grupo de trabajo debía leer el texto que llevaba por título la luz como productora de color, en la ejercitación se dispuso de un laboratorio que consistía en iluminar una esfera de color con diferentes rayos de luces de colores para que los estudiantes pudieran predecir o conjeturar el color que tomaría la esfera cada vez que incidían los rayos luminosos sobre ella, en la aplicación continuaron con el proceso de conjeturación y predicción mediante situaciones reales que suceden en los escenarios teatrales, finalmente en la complementación el alumnado compartía con su familia los aprendizajes adquiridos y construían un escrito a partir de la pregunta ¿cuál fue la importancia de los descubrimientos de Newton respecto a la naturaleza de la luz?. La fase experimental se desarrolló de manera particular en la casa de los estudiantes y se dispuso de 3 horas para su elaboración.

El desarrollo del material empleado para los laboratorios se dio a partir del uso y fabricación de instrumentos con materiales de fácil adquisición o presentes en el contexto cercano que permitieran simular algunas de las experiencias realizadas por Newton para formulación de su teoría. Algunas actividades debieron ser adaptadas a la contingencia generada por el Covid 19, respondiendo a las exigencias de distanciamiento social por parte de autoridades municipales. Por tanto se pensó en algunas otras que pudieran realizarse de forma individual en casa.

En cuanto al instrumento usado para conocer los avances en el proceso de aprendizaje del alumnado se dispuso un único cuestionario (pretest-postest), el cual fue aplicado antes y después de desarrollar la secuencia didáctica, el instrumento contenía 12 preguntas las cuales debían responder de acuerdo con una escala dicotómica $(\mathrm{Si}-\mathrm{No}$ ), además de justificar el porqué de cada una de las respuestas.

Los ítems fueron construidos de tal forma que evaluaran los aprendizajes desarrollados en cada una de las tres guías y se dispusieron en orden con el desarrollo de estas de tal manera que las 
preguntas 1 a la 4 trataban sobre la formación de la luz, las preguntas 5 hasta la 8 consideraban la clasificación de los colores de la luz, y las preguntas de los ítems 9 hasta el 12 se formularon de acuerdo con el tema de la mezcla de colores. Para el análisis de los resultados de cada uno de los cuestionarios se empleó una rúbrica (Ver tabla 3) de desempeños así: desempeño bajo (Bj), desempeño básico (Bs), desempeño alto (A) y desempeño superior (S), en la tabla 3

\subsection{Metodología de la sistematización y recolección de los datos}

La sistematización se realizó a partir de los instrumentos aplicados en cada momento de la intervención. Debido a la crisis sanitaria generada por el Covid 19 que condujo al Gobierno Nacional a tomar medidas de aislamiento social que evitaran la propagación del virus en el territorio nacional promulgado en la Resolución 385 del 12 de marzo del 2020, se tuvo la necesidad de

Institución Educativa El Madroño teniendo en cuenta lo anterior, realizó la indagación de los recursos dispuestos por los hogares y decidió implementar como mediación educativa, la red social WhatsApp por las siguientes razones:

1. El uso general por la mayor parte de la comunidad educativa.

2. La posibilidad que tiene de compartir documentos, imágenes, audios y textos.

3. Registra la conexión y desconexión de los estudiantes a las clases sincrónicas.

4. Las facilidades de uso.

5. Costo bajo para su utilización ya que se presenta incluido en la mayoría de los planes de datos (Prepago y Postpago)

De acuerdo con Meritxell, González y Guitert (2017) "El uso de la mensajería móvil y en este caso del WhatsApp, se consolida como una herramienta que facilita el trabajo colaborativo entre docentes y a su vez fomenta la creación y construcción conjunta de conocimiento curricular" (p. 5).

Con base en lo anterior, la aplicación de la propuesta investigativa se desarrolló durante el segundo semestre del año 2020, aún durante el aislamiento obligatorio. Se decidió usar la red social WhatsApp como mediación pedagógica para compartir los instrumentos diseñados, las orientaciones para su desarrollo, así como también para la recepción de las evidencias fotográficas, bibliográficas y audiovisuales de cada uno de los experimentos, las hipótesis y los aportes realizados por los estudiantes a lo largo de la propuesta.

FIGURA 1. Grupo de WhatsApp usado como mediación didáctica

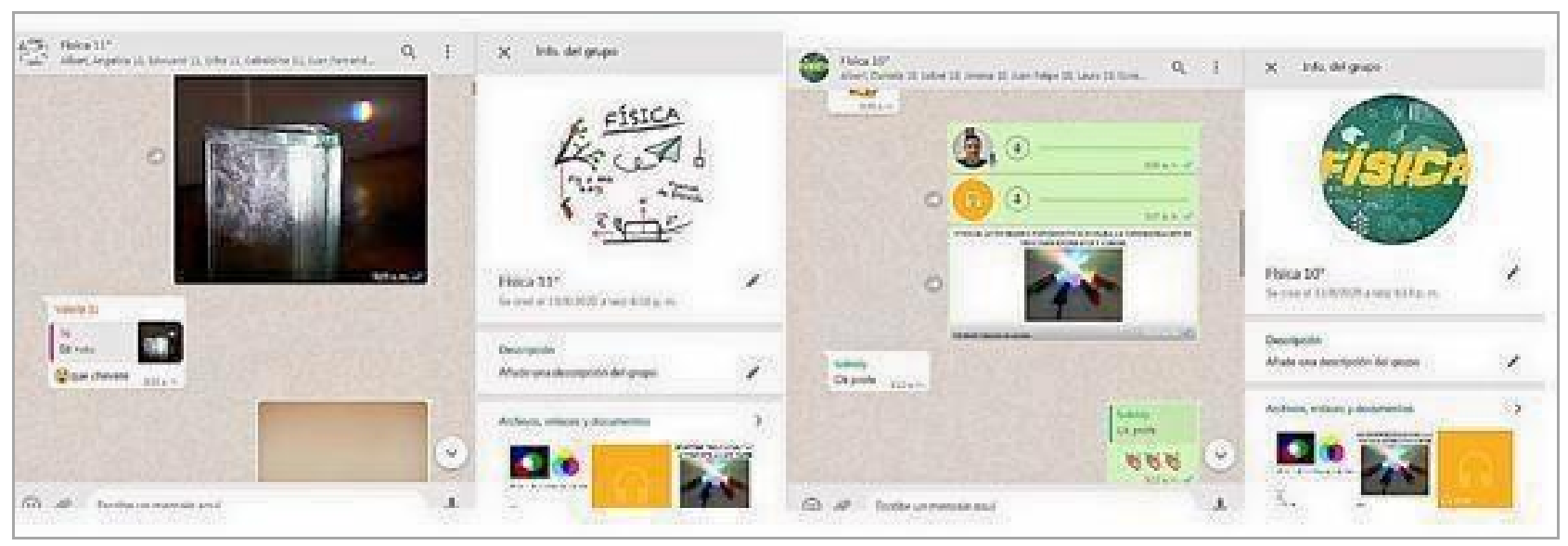

Fuente: elaboración propia 


\section{RESULTADOS}

En este apartado se muestran la prueba de validez de contenido de expertos y los respectivos análisis de las pruebas y actividades desarrolladas con el grupo de estudiantes.

\subsection{Prueba de validez de contenido por expertos}

Las tres guías de intervención fueron valoradas por un grupo de tres docentes expertos en el área de enseñanza de la física. Para ello se tuvo en cuenta su trayectoria docente e investigativa. El análisis de las guías se basó en cuatro tópicos:

1. El desarrollo de la guía lleva al estudiante a la comprensión del fenómeno que se desea intervenir.

2. La guía está bien diseñada, el lenguaje es claro, las preguntas son coherentes.

3. El objetivo de la guía está bien definido, y las instrucciones para su desarrollo son coherentes y claras.

4. La forma como están estructuradas las experiencias acerca del fenómeno de la luz, si se corresponden con la epistemología de la ciencia y su experimentación directa.

Para evaluar dichos tópicos se implementó una escala tipo Likert de frecuencias así: $1=$ muy en desacuerdo; 2 = algo en desacuerdo; 3 = ni de acuerdo/ ni en desacuerdo; 4 = algo de acuerdo; 5 = muy de acuerdo. La prueba de validez que se aplicó fue la prueba de coeficiente $\mathrm{W}$ de Kendall, que permitió decidir el nivel de concordancia entre los juicios de expertos, en la tabla 4, se puede apreciar el resultado obtenido.

TABLA 4. Prueba de validez de contenido por jueces expertos W de Kendall

\begin{tabular}{cc}
\hline \multicolumn{2}{c}{ Estadísticos de prueba } \\
\hline $\mathrm{N}$ & 36 \\
\hline W de Kendalla & 0,917 \\
\hline Chi-cuadrado & 33,000 \\
\hline $\mathrm{gl}$ & 1 \\
\hline Sig. asintótica & 0,000 \\
\hline
\end{tabular}

a. Coeficiente de

concordancia de Kendall

Fuente: elaboración propia realizada con el software SPSS v.21

De acuerdo con los resultados el valor W de Kendall es de 0,917 lo que indica una alta congruencia de análisis de las guías por parte de los expertos y por otra parte el nivel de significancia es de 0,000 este resultado en particular muestra que existe paridad, cohesión y coherencia entre los jueces para la validez del contenido de las guías.

\subsection{Análisis de la secuencia didáctica}

Debido a las medidas tomadas por la Secretaria de Educación del departamento de Caldas Colombia, se tuvo la necesidad de recurrir a nuevas dinámicas de enseñanza mediadas por la tecnología, así como a la disposición de cada estudiante por gestionar su aprendizaje. Por tanto, se decidió adaptar el material didáctico elaborado previamente ya que estaba pensado en conclusiones guiadas en el aula y construcción de conceptos a partir de la interacción del grupo. Para subsanar la dificultad, se recurrió a la creación de grupos de WhatsApp para la orientación, seguimiento y evaluación de la propuesta. Se envió por este medio, el consentimiento informado de uso de imagen de los estudiantes con el fin de que fuese aceptado por los acudientes, así como el formato de pretest y postest que fue desarrollado en casa y retornado a través de evidencia fotográfica. Ahora 
bien, se optó por la impresión de la secuencia didáctica para hacerla llegar de manera física junto con los elementos necesarios al momento de desarrollar los procesos experimentales.

Con base en las evidencias que fueron compartidas por los estudiantes y el desarrollo de la secuencia didáctica, se extraen algunas conclusiones que permiten intuir el nivel de apropiación del conocimiento generado durante a aplicación de la propuesta. Es de mencionar que el análisis también condujo a la necesidad de buscar material de apoyo como vídeos, texto o audios, que favorecieran la compresión de temas, reemplazando de cierta forma la mediación del docente en el aula de clase.

\section{Análisis de la guía 1. Formación de colores a partir de la luz blanca}

En el abordaje de cada uno de los momentos pedagógicos se intentó retomar los saberes previos de los estudiantes, estimular la creación de hipótesis, realizar actividades experimentales, formalizar los conceptos y trasladar el saber aprendido a los miembros de cada familia.

Uno de los instrumentos que tuvo que ser adaptado para permitir que pudieran ser replicadas las experiencias en casa, fue el prisma óptico. Debido a que los estudiantes no disponían de dicha herramienta, fue necesario recurrir a la elaboración artesanal de un prisma rectangular en vidrio que fue llenado con agua. Lo anterior permitió simular el efecto producido por el prisma óptico de manera que permitiera ser estudiado.

Inicialmente se optó por retomar los saberes previos relacionados con las mezclas de pigmentos. Lo anterior facilitó abordar la idea que existen colores primarios, que la mezcla de los mismos genera nuevos colores (secundarios) y que su comportamiento es diferente a los colores producidos por la luz.

FIGURA 2. Evidencias de actividades escolares sobre la formación de los colores

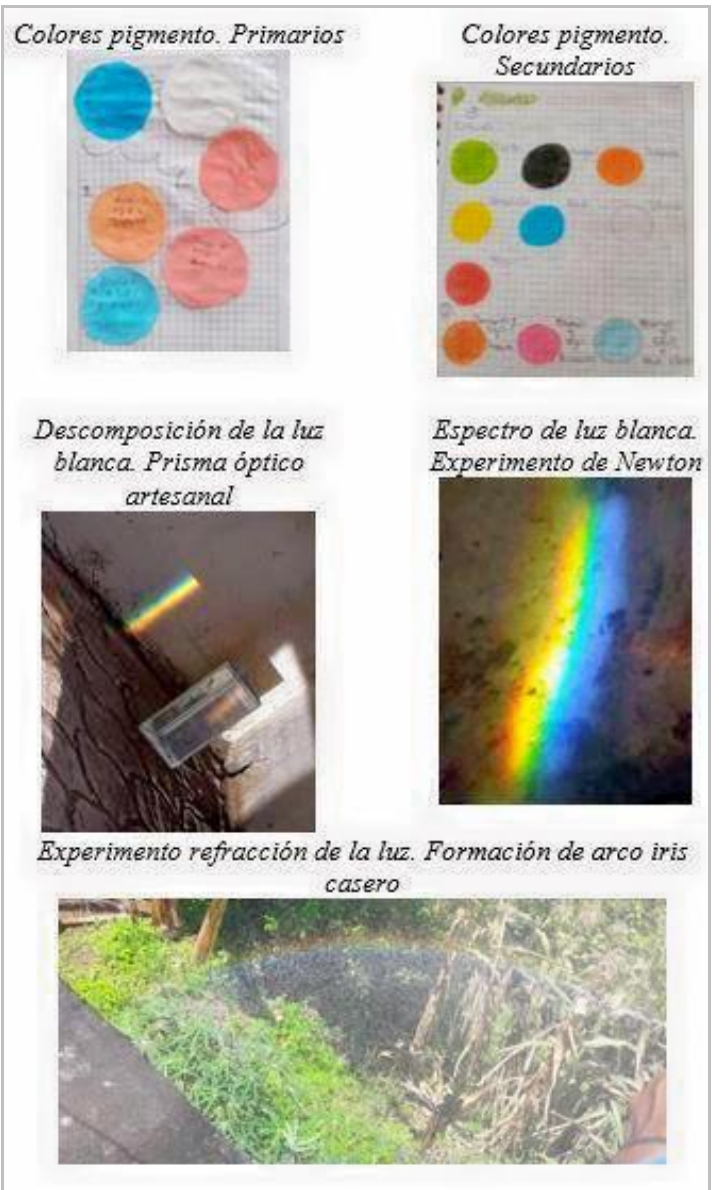

Fuente: elaboración propia con fotografías enviadas por los estudiantes 
Se resalta el compromiso mostrado por la mayoría de los estudiantes por aprender el comportamiento de la luz y la diferencia a su creación a través de pigmentos. Se percibió curiosidad por entender los fenómenos y se recurrió a diversas fuentes: video tutoriales y textos que fueron enviados por los educandos para complementar las explicaciones dadas por el docente. A pesar de ser una población rural, se utilizó material disponible en el medio como mangueras, tanques de almacenamiento de agua, espejos, linternas y celulares con el fin de simular algunas experiencias de la descomposición de la luz blanca y mezclas de colores, en la figura 2 se muestran algunas evidencias del desarrollo del experimento por parte de los estudiantes.

\section{Análisis de la guía 2. Clasificación de los colores de la luz}

En el desarrollo de la segunda guía se abordó la idea de cómo los colores prismáticos pueden ser separados nuevamente, para así establecer la noción de colores simples y compuestos. Se recurrió inicialmente a la realización de un experimento casero que usaba la luz solar y un espejo ubicado dentro de un recipiente con agua. La experiencia retomó la definición de luz conformada por diferentes colores.

Desde esta perspectiva, el experimento que Newton llevó a cabo da a conocer los criterios de corte experimental desde los cuales organiza los colores de la luz. Se considera que este experimento permite la construcción de una organización cromática en la que se definen cuáles colores de luces pueden producirse a partir de la mezcla de otros, y cuáles no; se trata de establecer los colores simples y compuestos de la mezcla de luces de colores. (Cepeda, 2017, p. 45)

Una de las dificultades marcadas para la configuración de vínculos entre el color y la luz fue la reproducción de la experiencia de Newton. La actividad experimental para establecer la noción de colores simples y compuestos fue difícil de replicar por los educandos ya que luego de separar la luz blanca en el espectro visible e intentar hacer pasar cada rayo de luz (color) de forma individual, por un segundo prisma se notó que al ser franjas delgadas de colores, producidas posiblemente por el uso del prisma artesanal no se distinguían los colores y por tanto fue necesaria la mediación del docente. Fue necesario reforzar la idea de que los colores primarios (simples) de la luz, corresponden al rojo, azul y verde y que partir de la combinación aditiva de los mismos se consiguen los demás.

FIGURA 3. Evidencias de actividades escolares sobre la formación de los colores

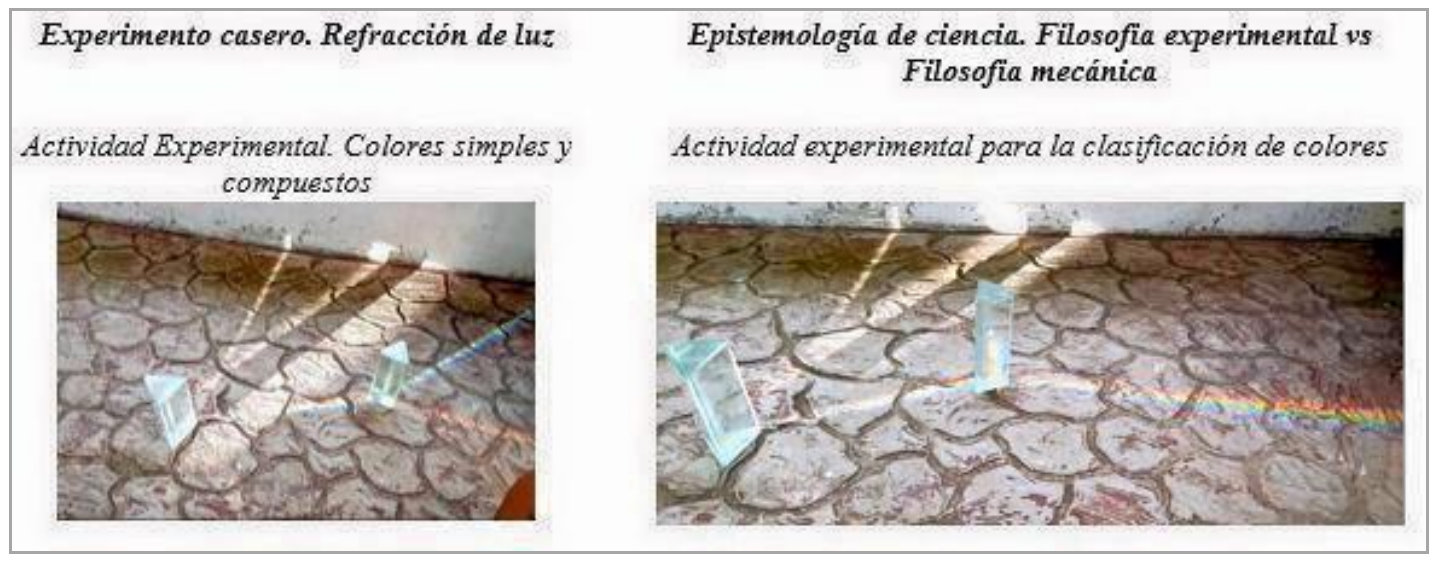

Fuente: elaboración propia con fotografías enviadas por los estudiantes 
Análisis de la guía 3. Mezclas de colores

El objetivo propuesto fue reconocer que el color es una cualidad de la luz y por tanto es manipulable, en consonancia con las ideas propuestas por Newton. Para ello se facilitó papel celofán con los colores simples (rojo, azul y verde). Se dio las orientaciones de usar linternas que serían interceptadas en la salida de luz por un papel de color, de manera que la proyectara.

Cabe destacar que un estudiante usó la misma linterna y simplemente fue sobreponiendo los papeles de manera que le permitiera observar el color de la luz proyectada. Lo anterior fue registrado en un vídeo y lleva a concluir que fue apropiada por el alumno quien modificó por sí mismo la instrucción, creando un ambiente diferente pero respetando la experiencia.

Así mismo, algunos estudiantes reemplazaron el uso de las linternas por teléfonos móviles, simulando la experiencia de la mezcla aditiva de colores

Debido a la cantidad de colores primarios, se genera una gran cantidad de colores compuestos, que difícilmente pueden diferenciarse. Además, pensando en la puesta en marcha de la propuesta en la escuela, esta organización sería poco productiva de acuerdo con los tiempos de trabajo de la intervención (Cepeda, 2017).

FIGURA 4. Evidencias de actividades escolares sobre mezclas de colores

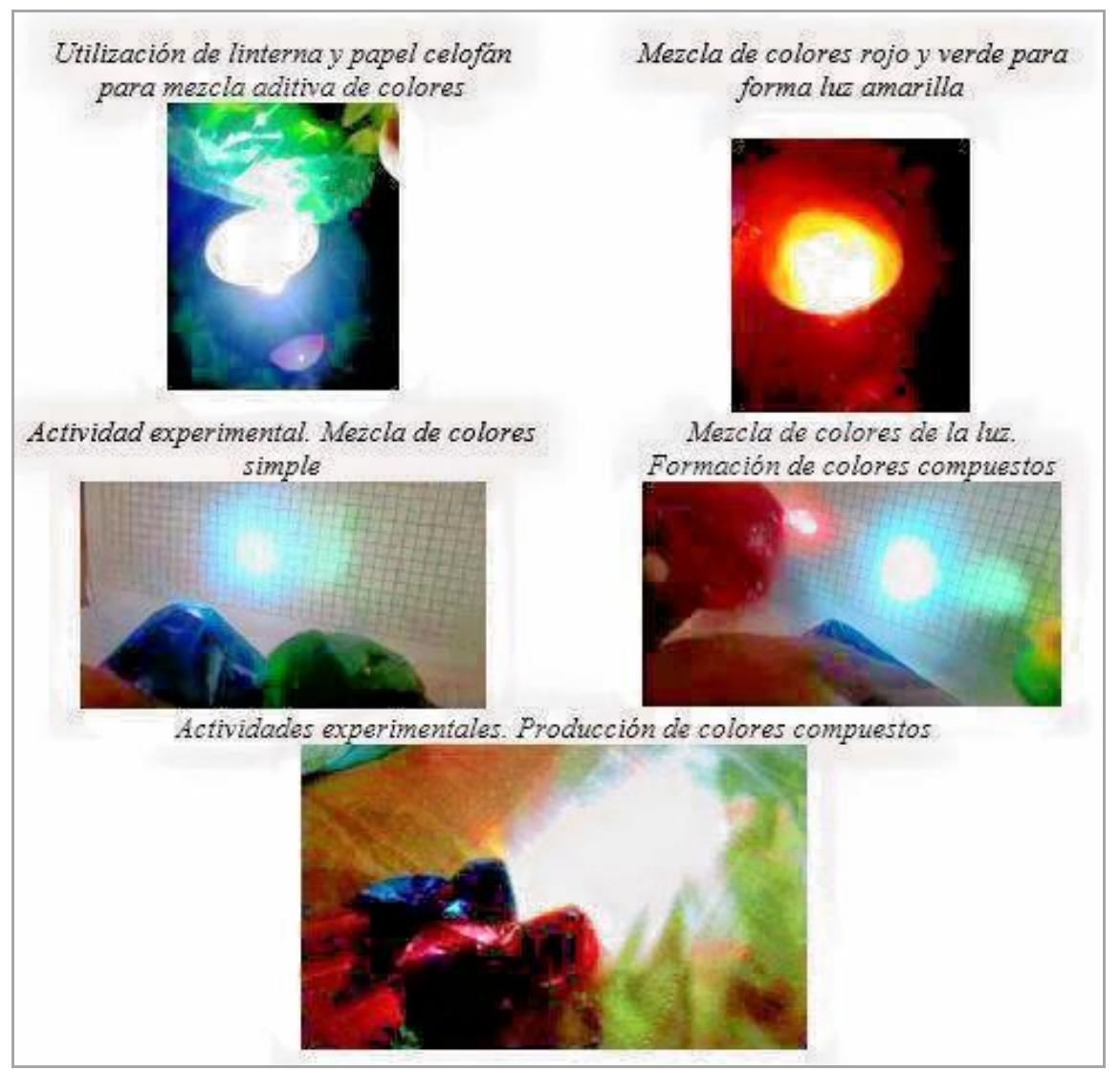

Fuente: elaboración propia con fotografías enviadas por los estudiantes

\subsection{Comparación y Análisis de los resultados del pretest contra el postest}

Antes de mostrar los resultados obtenidos, es importante aclarar algunos aspectos sobre el análisis descriptivo que se empleó en este trabajo. Si bien el enfoque que se utilizó es cualitativo, una buena forma de evidenciar el progreso en el aprendizaje de los conceptos sobre la teoría de la 
luz desde la metodología fenomenológica fue mostrar las frecuencias obtenidas en las dos pruebas por parte de los estudiantes. También se resalta que es una muestra pequeña $(n=15)$ y esto obedece a dos situaciones, en primer lugar el grupo experimental es intacto y por otro lado el contexto dónde se desarrolló esta investigación es de carácter rural, esta característica es importante definirla, ya que en Colombia las escuelas rurales tienen poblaciones con pocos estudiantes y este es el caso particular, además el proyecto se desarrolló en tiempos de pandemia, lo que no hizo posible tener una muestra mayor. También resulta muy significativo mencionar el trabajo desarrollado por los estudiantes en sus casas ya que involucraron de manera activa a sus familiares. En la figura 2, se pueden observar los resultados de las dos pruebas.

FIGURA 5. Resultados del pretest contra el postest

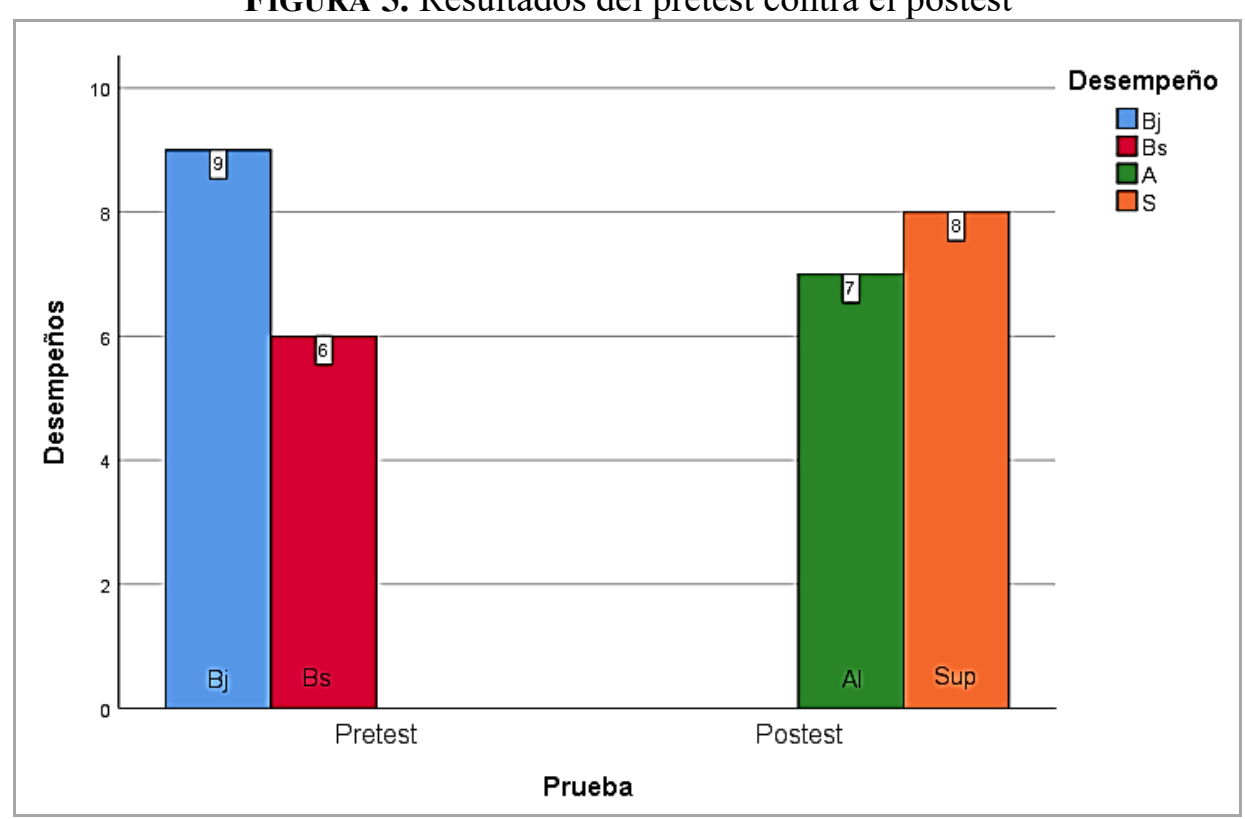

Fuente: elaboración propia realizada con el software SPSS v.21

Nota: Las abreviaturas: Bj indican los desempeños bajos, Bs indican los desempeños básicos, A indican los desempeños altos, y $\mathrm{S}$ indica los desempeños superiores

En la figura 5 se muestran los resultados obtenidos por el alumnado en la prueba pretest antes de ser aplicada la secuencia didáctica, y los datos del postest luego de ser realizada la misma. Al ser contrastados dichos resultados se puede notar la forma como mejoraron en los desempeños de la prueba postest ya que los estudiantes no obtuvieron ningún desempeño bajo (Bj) y Básico (Bs), por el contario alcanzaron 7 desempeños Altos (A) y 8 desempeños superiores $(\mathrm{S})$.

Lo anterior permite demostrar que el uso de la epistemología y la historia favorece la comprensión de los conceptos abordados por la teoría, en tanto, concede la facultad de discernir la razón que conduce a la ciencia a la aceptación del modelo actual al ser comparado con otros estudios previos. Además, presenta el experimento como un instrumento que admite la construcción de conocimiento científico ya que ayuda al aprendiz a generar hipótesis antes, durante y después de aplicado el ensayo, que serán juzgadas a partir de la experiencia y de las que podrán formalizar, demostrar o construir nuevos saberes.

Esto se puede evidenciar en los resultados de algunos trabajos desarrollados por Furió-Más, Furió-Gómez y Solbes (2012); y Adúriz-Bravo (2013), en los que se destaca ampliamente la necesidad de que los maestros cada vez se formen más y aprendan acerca de la historia y la epistemología para fortalecer el proceso de aprendizaje y a la vez sirvan para la alfabetización en ciencias de los estudiantes. 


\section{DiSCUSIÓN}

Este trabajo de investigación partió de la pregunta problematizadora: ¿Cómo fortalecer la enseñanza y el aprendizaje de la teoría de la luz del siglo XVII propuesta por Isaac Newton en educación media? Dicho interrogante llevó a la búsqueda de una metodología en la que se vivenciara el proceso por parte de los estudiantes y para ello se recurrió al uso de la experimentación mediada con la historia y la epistemología de la ciencia. De acuerdo con Furió y Guisasola (1997) "Una de las aportaciones de la historia y la epistemología de la ciencia es que nos permite establecer las características fundamentales de la naturaleza de la ciencia y el trabajo científico" (p. 260), esta situación se pudo evidenciar durante el desarrollo de esta propuesta ya que los estudiantes identificaron los principios de la descomposición de la luz a través del prisma óptico de la misma manera que lo hizo Newton. Además se percataron de las vicisitudes por las que debió pasar el científico para poder formular una teoría, así mismo el experimento fortaleció el aprendizaje ya que permitió ver de forma clara lo que sucede en dicho instante que se proyecta. Por otro lado es vital que en la enseñanza de las ciencias se logren integrar tanto conceptos, procedimientos como principios epistemológicos a través de un enfoque didáctico apropiado (Solbes, 2011), lo cual no ha sido la realidad histórica mostrada (Flores, Caballero y Moreira, 2009).

De acuerdo con los resultados obtenidos en la prueba del postest son muy significativos los desempeños de los estudiantes, posterior al desarrollo del instrumento de intervención, esto se debe en parte a la forma como fueron abordados los conceptos de la teoría de la luz, ya que permitió a los estudiantes interactuar con la experiencia a partir del desarrollo histórico.

Así mismo, se resalta como la pandemia no fue un impedimento para el desarrollo del trabajo en casa, por el contrario se pudo notar la ayuda de los familiares, además la red social WhatsApp, se convirtió en una herramienta fundamental para la socialización de los trabajos, puesto que en el grupo que se dispuso la comunicación, este se convirtió en un foro de discusión que facilitaba la explicación de las guías.

Finalmente una de las dificultades que se pudo evidenciar, obedece a la falta de conectividad de los estudiantes, lo que dificultó la recolección de la información, no obstante se logró en un porcentaje favorable contar con los trabajos desarrollados en casa.

\section{Conclusiones}

La interpretación de la experiencia de Newton ha permitido construir un método de enseñanza de conceptos relacionados con la naturaleza la luz y la formación de los colores a partir de un enfoque epistemológico que facilita el entendimiento de las controversias generadas por las misma en el siglo XVII y la aplicación de procedimientos experimentales para la construcción de conceptos previos a la formalización de la teoría, propiciando la formulación de hipótesis y conjeturas, elementos esenciales para el fortalecimiento del pensamiento científico.

Se percibe que al finalizar la propuesta investigativa los estudiantes de educación media de la institución educativa lograron identificar conceptualizaciones de la naturaleza de la luz y la formación de los colores a partir de un enfoque epistemológico y experimental generando de esta manera un aprendizaje significativo.

La realización de la investigación permitió despertar en los estudiantes el interés y la motivación por el aprendizaje de la física a partir de la historia y la experimentación aplicada a la teoría de la luz de Newton en siglo XVII.

El trabajo colaborativo fue de vital importancia en el desarrollo de cada una de las etapas del proceso investigativo permitiendo mejorar las relaciones interpersonales de los estudiantes con sus familias, sobrellevando así, la contingencia generada por el Covid 19. Por otra parte, la mediación pedagógica a través de WhatsApp permitió generar un ambiente de aprendizaje que integró 
diferentes actores y elementos de la comunidad educativa, demostrando que es posible el aprendizaje desde casa.

Se diseñó una estrategia didáctica que permitió la comprensión de los modelos de la luz del siglo XVII. Permitiendo la humanización de las ciencias exactas a partir del conocimiento de la historia. Dicho instrumento fue evaluado en términos del conocimiento construidos por los estudiantes a partir del análisis estadístico de los resultados obtenidos en el postest

\section{Declaración de conflicto de intereses}

Los autores manifestamos que durante la ejecución de este trabajo y su redacción, no han incidido intereses personales o ajenos a nuestra voluntad, donde se puedan incluir malas conductas y valores distintos a los que usual y éticamente tiene la investigación.

\section{Referencias}

Adúriz-Bravo, A. (2013). La historia de la ciencia en la enseñanza de la naturaleza de la ciencia: Maria Skłodowska-Curie y la radiactividad. Educació Química, 10-16. Recuperado de https://bit.ly/2SC2FVA

Aguilar Rodríguez, Y., y Alamino Ortega, D. de J. (2014). Integración de la Historia y la Filosofía de la Ciencia y la enseñanza de la física: Fundamentaciones y experiencias en Cuba The history of physics and epistemology, in phisics teaching View project. III conferencia latinoamericana del international, history and philosophy of science teaching group IHPST, 1-12. Santiago de Chile.

Álvarez, E., y Manzano Diosdado, D. (2018). Propuesta didáctica para el empleo de la Historia de la Ciencia en la Enseñanza del primer Principio de la Termodinámica en Educación Secundaria. Revista de Educación de La Universidad de Granada, 25, 9-28. Recuperado de https://bit.ly/34oZVxu

Bisquerra Alzina, R. (2009). Metodología de la Investigación Educativa. La Muralla.,S.A.

Bravo, S. del V. (2016). La experimentación en el aprendizaje de la física: su incidencia en la construcción de conceptos referidos a la óptica ondulatoria. [Tesis de maestría]. Universidad Nacional del Centro de la Provincia de Buenos Aires.

Castro García, E., Gómez Fernández, P., y Llavona Díaz, L. (2012). La historia de la ciencia como recurso didáctico en Física y Química desde un punto de vista constructivista. Tiempo y Sociedad, 8, 68-88

Cepeda Hernández, N. A. (2017). La construcción de vínculos entre luz y color desde la perspectiva de Newton: Una propuesta para ampliar el campo de los fenómenos cromáticos. [Tesis de maestría]. Universidad Pedagógica Nacional de Colombia.

Flores, J., Caballero Sahelices, M. C., y Moreira, M. A. (2009). El laboratorio en la enseñanza de las ciencias: Una visión integral en este complejo ambiente de aprendizaje. Revista de Investigación, 33(68). Recuperado de https://bit.ly/3fu9sK0

Fundación Escuela Nueva. (2021). Fundación Escuela Nueva. Volvamos a la gente. Recuperado de https://escuelanueva.org/

Furió-Más, C., Furió-Gómez, C., y Solbes-Matarredona, J. (2012). Profundizando en la educación científica: aspectos epistemológicos y metodológicos a tener en cuenta en la enseñanza. Educar Em Revista, 44, 37-57. DOI:10.1590/S0104-40602012000200004

Furió, C., y Guisasola, J. (1997). Historia y epistemología de las ciencias deficiencias epistemológicas en la enseñanza habitual de los conceptos de campo y potencial eléctrico. Enseñanza de Las Ciencias, 15(2), 259-271. Recuperado de https://bit.ly/3yKfeii

Íñiguez Rueda, L. (1999). Investigación y evaluación cualitativa: bases teóricas y conceptuales. Atención Primaria, 23. Recuperado de https://bit.ly/3c1cC61

Lafuente, A., Valverde, N., y Pimentel, J. (2004). El Telescopio de refelexión. Newton entre luces y 
cristales. Consejo Superior de Investigaciones Científicas (España).

López Monsalve, C. D. (2020). Relación de los proyectos extracurriculares con las orientaciones motivacionales y las estrategias de aprendizaje, una apuesta por los ambientes pedagógicos en el marco del modelo Escuela Nueva [Tesis de maestría]. Universidad Católica de Manizales.

Matthews, M. R. (1991). Un lugar para la historia y la filosofía en la enseñanza de las ciencias. Comunicación, Lenguaje y Educación, 3(11-12), 141-156. DOI:10.1080/02147033.1991.10820987

Meritxell Monguillot, H., González Arévalo, C.,y Guitert Catasús, M. (2017). El whatsapp como herramienta para la colaboración docente. EmásF: Revista Digital de Educación Física, 44, 56-62. Recuperado de https://bit.ly/3ftivL3

Monroy Rivera, A. M. (2016). La experimentación como estrategia para la enseñanza aprendizaje del concepto de materia y sus estados [Tesis de maestría]. Universidad Pedagógica Nacional de Colombia.

Morcillo Molina, C. (2016). La experimentación en la enseñanza de las ciencias para docentes en formación inicial: un caso en microbiología. Una mirada desde la historia de las ciencias. [Tesis de pregrado]. Universidad del Valle.

Perales Palacios, F. J., y Nievas Cazorla, F. (1991). Ideas previas en óptica geométrica: un estudio descriptivo. Revista Investigación En La Escuela, 13, 77-84. Recuperado de https://bit.ly/3i0WW6r

Pérez Lozada, E., y Falcón, N. (2009). Diseño de prototipos experimentales orientados al aprendizaje de la optica. Revista Eureka Sobre Enseñanza y Divulgación de Las Ciencias, 6(3), 452-465. Recuperado de https://bit.ly/3frnhsG

Solbes, J., y Sinarcas, V. (2009). Utilizando la historia de la ciencia en la enseñanza de los conceptos claves de la física cuántica. Didáctica de las Ciencias Experimentales y Sociales, 23, 123-151.

Solbes, J. (2011). Indagación sobre la visión. En M. del P. Jiménez Alexandre (Ed.), Cuaderno de indagación en el aula y competencia científica (pp. 103-121). Colección Aulas de Verano. Recuperado de https://bit.ly/3yM0Ohx

Solbes, J., y Traver, M. J. (1996). La utilización de la historia de las ciencias en la enseñanza de la física y la química. Enseñanza de las ciencias: revista de investigación y experiencias didácticas, 14, 103-112.

Solbes, J. y Traver, M. (2001). Resultados obtenidos introduciendo historia de la ciencia en las clases de física y química: mejora de la imagen de la ciencia y desarrollo de actitudes positivas. Enseñanza de Las Ciencias: Revista de Investigación y Experiencias Didácticas, 19(1), 151-162.

Solbes, J. y Traver, M. (2003). Against a Negative Image of Science: History of Science and the Teaching of Physics and Chemistry. Science \& Education, 12(7), 703-717. DOI: 10.1023/A:1025660420721

Vargas-Vargas, S. J., y Carmona-Ramírez, L.H. (2021). Enfoque epistemológico y experimental i en la enseñanza de las ciencias, una estrategia didáctica para el aprendizaje de la teoría de la luz de Newton. Didáctica de las Ciencias Experimentales y Sociales, 41, 155-170. DOI: 10.7203/DCES.41.20806 
